\title{
Urgences
}

\section{Fragments, journaux, carnets : prendre tout en note, noter le rien}

\section{Ginette Michaud}

Numéro 31, mars 1991

Poétique de la note

URI : https://id.erudit.org/iderudit/025637ar

DOI : https://doi.org/10.7202/025637ar

Aller au sommaire du numéro

Éditeur(s)

Urgences

ISSN

0226-9554 (imprimé)

1927-3924 (numérique)

Découvrir la revue

Citer cet article

Michaud, G. (1991). Fragments, journaux, carnets : prendre tout en note, noter

le rien. Urgences, (31), 67-84. https://doi.org/10.7202/025637ar d'utilisation que vous pouvez consulter en ligne.

https://apropos.erudit.org/fr/usagers/politique-dutilisation/ 


\section{Fragments, journaux, carnets: prendre tout en note, noter le rien Ginette Michaud}

Que je suis loin de la sincérité parfaite, et comme j'ai envie et peur, tout à la fois, de l'effort - considérable - qu'il faudrait pour j'allais dire tout dire, mais c'est impossible -, pour dire tout ce à quoi j'attache, moi, de l'importance sans me soucier d'un fantôme de lecteur. Ó Stendhal écrivant: On verra bien que ces notes ne sont que pour moi. Quel fantôme de lecteur regardait par-dessus son épaule?

\section{Octave Mannoni, Nous nous quittons. C'est là ma route}

Dans son excellent essai consacré au genre de la correspondance, L'équivoque épistolaire, Vincent Kaufmann fait remarquer à juste titre combien «le statut des écrits intimes ou "personnels" est souvent plus trouble qu'il n'y paraît" ${ }^{1}$. Les différents usages du registre épistolaire qu'il analyse à partir des correspondances des écrivains de la modernité (Baudelaire, Mallarmé, Proust, Flaubert, Rilke, Kafka, Artaud, entre autres) sous leur rapport ambigu, sinon pervers, à la Loi et à ses représentants (le symbolique, la langue, la mère, l'Autre), souscrivent tous à des degrés divers à une fondamentale équivoque dont on pourrait traduire ainsi la logique hyperbolique: plus les lettres se multiplient et se dépensent en direction d'une (apparente) communication et d'une (souhaitée) proximité avec l'autre, plus elles creusent de fait l'écart, l'irréductible distance entre les correspondants; plus les lettres semblent s'adresser à un interlocuteur unique, plus elles visent, au-delà de celui-ci, un destinataire tout d'ombre, une figure du vide, pure image qui ruine toute possibilité réelle d'échange et tente de liquider, souvent littéralement, le récepteur (cette mise à mort symbolique touche surtout des femmes, mères et amoureuses) à qui ces lettres sont adressées.

1 Vincent Kaufmann, L'équivoque épistolaire, coll. "Critique ", Paris, Minuit, 1990, p. 176. 
68

Si je rappelle les grandes lignes de l'hypothèse soulevee par Vincent Kaufmann au sujet de la correspondance, c'est qu'elle éclaire en retour celle que j'aimerais aborder ici, dans le cas des notes écrites "pour moi " seul, du moins à première vue, ces notes où l'adresse prend la forme particulière du retour à l'envoyeur, mais au prix de détours plus tortueux, en tout cas plus tourmentés, qu'il n'y parait.

Comme les lettres, mais un peu différemment d'elles, les fragments, esquisses, associations, réflexions et notes diverses, qu'ils soient laissés à l'état de paperoles ou recueillis dans des carnets et des journaux, fascinent en effet souvent le lecteur parce qu'ils appartiennent eux aussi à cette «zone énigmatique [...] où la vie passe parfois dans une œuvre, et inversement ${ }^{2}$ et qu'ils font partie de cette classe d'écrits mais s'agit-il encore d'un genre? Rien n'est moins sûr - où la "vie» et l'" œuvre» n'ont jamais paru plus proches, mais se révèlent tout compte fait éloignées de la plus irréparable manière. Tout lecteur de journal intime aura fait l'expérience de cette déception: croyant enfin tenir l'Auteur, il s'aperçoit que la "sincérité", la "vérité», l'« authenticité", les faits bruts, bref, tous ces attributs d'un sujet mis à nu dans sa parole la plus "personnelle", ne sont en réalité qu'illusion, masque arraché qui laisse apparaître un autre masque, jamais le visage. De fait, le malentendu évoqué par Kaufmann est encore aggravé par ces « fragments de vie trop écrits pour les uns, textes trop peu textuels pour les autres» qu'on aurait aimé refouler du domaine de la grande littérature parce que «trop prosaïques, voire franchement tri[viaux]» 3 . Comment justifier la lecture des notes de blanchisserie de Nietzsche, des rendez-vous, pris pour être manqués, de Proust, des dettes de l'un, des conquêtes amoureuses de l'autre? La question est sans doute mal posée et devrait plutôt s'entendre de la façon suivante: comment résister à la curiosité de tout lire? Où tirer le trait devant cette masse de matériaux, des déchets certes, mais ô combien révélateurs, qui viennent capter - captiver la lecture par un détail, un relief, un rien où se laisse soudain lire l'affect, telle l'empreinte du fossile dans la pierre?

La note, son traitement par le lecteur, implique, plus qu'un autre genre peut-être, la définition d'une éthique qui commence 
seulement de s'esquisser. Depuis une vingtaine d'années, la critique génétique a remis à l'honneur l'étude de ces écrits intimes, elle a déployé autour de ces textes marginaux et périphériques des questions et des moyens techniques impressionnants qui visaient à leur assurer un statut scientifique (ils étaient le «laboratoire » de l'œuvre) et à accréditer leur institutionnalisation au sein des études littéraires. Le consensus est désormais acquis, mais cette critique a-t-elle vraiment réussi, en dépit de tous ses efforts, à respecter la fragilité intrinsèque de ces écrits qui par nature font échec à toute poétique le moindrement systématique? Les notes, en s'accumulant, finissent en effet parfois par faire œuvre, mais sur un mode mineur, un mode qui mine et défait l'œuvre qui se développe ailleurs et parallèlement dans le temps, comme sur une autre planète. Par ailleurs, cette critique a-t-elle suffisamment pris la mesure du scandale au cœur de cette situation de lecture lorsqu'elle s'est empressée de légitimer (pour ensuite mieux l'oblitérer) ce "fantôme de lecteur" qui regarde par-dessus l'épaule de l'écrivain? Car il ne s'agit plus seulement d'indiscrétion, de voyeurisme, ou de curiosité déplacée, comme on dit couramment (bien qu'il y ait toujours regard à la dérobée, coup d'œil, dès qu'on lit les notes de quelqu'un d'autre). Stendhal l'avait bien vu: l'essentiel du paradoxal contrat - le plus souvent posthume, donc testamentaire: la note renvoie toujours à plus tard, elle ne vaut que pour la relecture qu'elle anticipe -, à l'œuvre dans les journaux et carnets, tourne autour de cet équivoque lecteur. On tient un journal pour que "soi " soit lu comme un autre, pour reprendre le beau titre du dernier ouvrage de Paul Riccur, mais plus encore peut-être pour que l'autre (ce «On verra " de la rumeur de la postérité, qui rend la phrase de Stendhal si paradoxale) retourne enfin ces notes au compte du seul moi ("ces notes ne sont que pour moi": le "ne... que» dit assez clairement qu'elles ne le sont jamais clairement à l'origine).

Plutôt que de tenter une véritable poétique de la note, j'aimerais seulement circonscrire par ces quelques propositions, elles-mêmes mimétiquement déliées, quelques-uns des effets produits par l'accumulation des notes, "sans tri et sans apprêt " 4 , à partir de pratiques différentes, chaque fois singulières, où la note devient poétique.

4 Octave Mannoni, "L'espace analytique ", NOus nous quittons. C'est là ma route. Carnets, Paris, Denoël, 1990, p. 75 . Les références à cet ouvrage seront dorénavant indiquées directement dans le texte. 
D'emblée, la publication du journal d'un psychanalyste aussi connu qu'Octave Mannoni - il s'agit de fait de trois Carnets écrits de 1921 à 1958, couvrant donc une période de près de quarante ans - piquera l'intérêt du lecteur, ne seraitce que par le fait que Mannoni fut l'un des premiers analysés de Lacan et qu'il resta lui-même, tout au long de sa brillante carrière (la "vraie vie" commença pour lui assez tardivement, à quarante ans, et ce fait n'est pas tout à fait étranger à l'abandon de ce journal), un écrivain et un psychanalyste des plus originaux. Publiés de façon posthume, ces Carnets n'ont cependant pas été livrés tels quels, ils ont subi un double toilettage éditorial: d'une part, tous les trivia en ont été supprimés (écartés «les fragments s'apparentant aux notations d'un agenda ou d'un répertoire: index des noms du second volume, indications de lecture, citations isolées, note du quotidien, annonces de rendez-vous, listes d'adresses, invitations..." [p. 5]); d'autre part, l'inévitable "discrétion familiale» a également exigé son dû et sa part d'omissions. Sans invoquer la censure - ce qui serait exagéré dans les circonstances: on peut en effet aisément comprendre que certaines précautions aient été prises pour protéger les personnes mises en cause par certaines notes intempestives du journal -, les Carnets ont donc néanmoins fait l'objet d'une haute surveillance, et cette "Note de l'éditeur » fait déjà à elle seule rêver le lecteur. Car si l'on tient qu'un journal et à plus forte raison lorsqu'il est le lieu, comme dans le cas de Mannoni, d'une double venue à l'écriture et à la psychanalyse - constitue une pratique qui présente quelque analogie avec la situation psychanalytique dans la mesure où l'on y observera la règle de noter tout ce qui vient à l'esprit selon "le mouvement difficile de la pensée libre» (p. 75), le geste qui consiste à expurger les Carnets des "indications de lecture", "citations isolées " et autres «notes du quotidien" et à ainsi opérer un tri parmi les informations dignes de figurer dans un "cahier de brouillon" qui se donnait lui-même "sans tri et sans apprêt", s'avère éminemment plus problématique. Quels critères permettront en effet de justifier le retrait de ces notes des Carnets, dont la visée était précisément de les enregistrer? Tout au long du journal, Mannoni apparaît luimême souvent préoccupé par les limites de l'entreprise, et s'exerce à mesurer de manière réflexive la portée de la note, en soulignant impérativement par exemple le devoir auquel 
elle ne peut, bien entendu, que manquer, comme dans cette liste de règles qu'il se prescrit régulièrement: «6) Tenir un journal avec plus de soin et y faire entrer en détail les questions professionnelles. Souvent, quand je ne les note pas, je m'aperçois que c'est parce que je n'en suis pas satisfait. C'est un tort. Noter!» (p. 260). Ailleurs, il s'imposera l'exercice (scolaire) de la note du quotidien: «Essayons de noter une journée avec quelques détails» (p. 187), comme pour éprouver, à nouveau, l'impossibilité de la tâche. De même, pour les indications de lecture, jamais assez nombreuses ou détaillées, semble-t-il, comme en témoigne cette autre liste de choses à faire: «H. [...] Lire, avec des notes de lecture, le plus possible» (p. 313). Une note autocritique commente en après-coup, en bas de page: «Pas tenu ce programme, comme j'aurais dû (15 octobre)."

Cela dit, en dépit donc des notes qui en auront été retirées (et que le lecteur supposera toujours plus intéressante que celles qui lui sont données à lire), les Carnets de Mannoni sont passionnants pour plusieurs raisons (ce qui ne veut pas dire que leur lecture soit pour autant exempte de malaise: ce n'est pas, après tout, le Mannoni auquel on est habitué qu'on rencontrera ici, mais un autre - tourmenté, jaloux, obsessionnel, sombre, etc. - appartenant à une vie antérieure, non encore transformée par l'analyse et l'écriture). Ainsi, du point de vue qui me retient ici, on sera frappé par le fait que, à travers la variété, somme toute moins grande qu'on pourrait le penser, de sujets abordés dans ce journal - spéculations philosophiques et poèmes du jeune homme, hésitations de la vie amoureuse, instabilité de la vie professionnelle, réflexions sur l'expérience coloniale de Madagascar, puis importante croissante de la notation des rêves, qui soulève en elle-même un problème $e^{5}-$, une

5 Sur les problèmes soulevés par la notation des rêves, qui occupent une grande part de ce journal, on lira le passage suivant: “ Je viens de relire, je n'ai pas rendu l'impression exacte, mais une autre un peu différente peut-être, et j'ai brodé un peu trop pour la retrouver, mais inutilement. Maintenant, c'est tout à fait fini... L'impression était très touchante, indiciblement, mais aussi plus absurde et fantastique que je n'ai su la rendre" (ibid., p. 242); ou encore celui-ci, observation faite une fois l'analyse commencée: “ Je n'ai jamais noté un type de rêve qui revient. Je ne l'ai pas noté parce qu'il est toujours informe (ibid., p. 273). La fugacité du rêve, ses détails qui s'estompent si rapidement, son caractère informe renvoient aux limites de la note elle-même: comme l'écrit Mannoni, « [c]ertaines choses sont à peine vraies, tant elles sont fugitives. J'ai 
réflexion sur la pratique de la note traverse en pointillé ce journal et lui sert même de point de repère, si ce n'est de fil d'Ariane. Les retours critiques sur la note per se s'intensifient en effet tout au long du journal, tout particulièrement à la fin du premier carnet qui couvre la plus longue période (19211944), et qui se clôt sur la découverte d'une adresse fondamentalement ambiguë de cette écriture (p. 125):

Je m'intéresse davantage à ce journal, avoue Mannoni, depuis que $v$ l'a découvert. Ainsi, dans mon inconscient, il se peut que je ne l'écrive pas tout à fait pour moi seul. Tant pis. Mais cela montre qu'il faut aller plus loin dans la sincérité. Je suis tout à fait à la surface.

À partir de ce moment, les choses vont se précipiter: le deuxième Carnet se concentre sur seulement deux années, décisives à plus d'un titre, 1944-1945: l'entrée en analyse, avec dédoublement momentané du journal en un cahier d'analyse pour une très brève période (du 13 novembre au 3 décembre 1945, trois semaines à peine), se produit presque à la toute fin de ce second carnet, et elle entraîne dans le troisième carnet une réflexion de plus en plus nourrie sur la fonction du journal, en même temps qu'une interrogation radicale sur la difficulté grandissante de l'objet à noter, qui se solderont par la désintégration, la dissolution finale du journal en 1958. Désormais, l'écriture et l'analyse absorberont l'énergie qui était mise en réserve, mobilisée pourrait-on dire, par le journal et son entreprise de contrôle du "réel ", surtout sous la forme d'un style télégraphique qui cherchait à le saturer en le prenant de vitesse.

Car tout en croyant d'abord tenir son journal à des fins mnémotechniques:

Chez moi, tenir un journal est une grande aide - car j'ai vraiment peu de mémoire. Mais je n'y note guère ce que je suis sûr de me rappeler. Au fond, j'y inscris: 1) ce que je ne veux pas oublier; 2) ce que je veux essayer de débrouiller pour moi seul. Et aussi, je m'apercevrais de ce que mon humeur et mes opinions ont de "labile " si je ne les fixais pas (p. 172),

tort de ne pas les noter, et de les négliger à cause de ce caractère douteux. Je devrais au contraire m'y attacher " (ibid., p. 268): mais justement, la note, comme le réve, fuit. 
c'est bien plutôt cette expérience déstabilisante d'une labilité, d'une inconstance et d'une inconsistance fondamentales du moi que Mannoni va découvrir au fil de ces notes décousues. Source de "mécontentement de soi " (p. 321) inépuisable, la note va, de récriminations en protestations, de plaintes en déceptions, agiter et exacerber la pseudo-solidité du moi, elle va le fatiguer, l'épuiser jusqu'au désaisissement. Noter des riens fait ainsi découvrir, littéralement, le Rien, ces incidents du réel qui font trébucher le sujet.

Aspirant à un pur décrire («[...] ces notes sont idiotes. Je devrais décrire et non juger " [p. 244], dépourvue de sensations et d'impressions, cherchant à " éviter tout romantisme et tout lyrisme» [p. 337]), la note chez Mannoni est constamment vouée à l'échec, coincée dans une double contrainte, oscillant entre le «trop" et le " pas assez": "Le plus mauvais, dans ce que j'écris, c'est ce qui est tant soit peu littéraire ou théorique. Le meilleur, ce sont les notations de faits personnels observés - sans trop d'analyse. Mais je ne les raconte guère" (p. 173). Autrement dit, le meilleur, c'est toujours paradoxalement la note qui manque, le détail qui n'est pas raconté, les rêves qui ne sont pas "notés à temps" (p. 287) et qui échappent de justesse à la transcription ( $\mathrm{Au}$ réveil, souvenir de rêves confus que je n'aurais pas noté (si je n'obéissais au $\mathrm{D}^{\mathrm{r}}$ Lacan)» (p. 273). La plainte, inlassable, revient: «Je devrais noter dans ce cahier les idées qui me viennent. [...] (Écrit plus tard: Quel tort de ne pas le noter!)." (p. 338). Chargée de tout dire, la note finit par ne plus rien dire, mieux, elle finit par être l'écriture du rien; indexée comme point de repère de la mémoire défaillante, elle finit par tout oublier, mais elle n'oublie pas qu'elle oublie, selon un mouvement qui met toujours le diariste dans son tort, qui le rend coupable de n'avoir pas su d'avance où couper avec discernement dans la masse des choses qui se présentaient à lui. Déjà, au tout début du journal, après un an seulement, cette forme d'écriture faisait tout à la fois l'objet d'un rejet et d'une défense, elle était préservée pour être révoquée:

II y a un an que j'ai commencé ce journal. II n'est guère intéressant. Cependant, tel qu'il est, je le continuerai si je peux. Si je croyais que la chose en valût la peine, je ne ferais pas ainsi car j'ai trouvé la bonne méthode, que je n'appliquerai pas. Ce serait non pas de faire un journal, mais de prendre tout en note, les moindres impressions, les noms propres, les adresses, les dates, les jours de pluie. Et alors, à 
74

la fin de l'année, qu'il serait beau de tout ce fouillis, avec un calendrier "pour savoir quand il y eut de la lune et quand il n'y en eut pas", de refaire un journal bien écrit, et, chose impossible autrement, bien composé. Seulement, je n'écris que pour mol. Si j'avais fait ainsi, que de choses j'aurais aujourd'hui à raconter, qui ne sont point dans ce carnet (p. 73).

"La bonne méthode que je n'appliquerai pas»: on ne saurait mieux dire que la note manquera toujours de méthode pour bien découper le réel, mais aussi que le projet de «tout dire" doit nécessairement s'inscrire dans la note manquante s'il veut échapper au discours délirant. Ce n'est pas un hasard si c'est ce genre de note inscrivant le manque précisément en le laissant vide, qui devient le leitmotiv le plus caractéristique du journal de Mannoni, le scandant à intervalles réguliers, fût-ce sous la forme la plus banale du regret: "Terriblement négligé ce journal, à tort. J'aurais quantité de choses à y noter. Je vais tâcher d'y revenir» (p. 303). Il n'est même plus nécessaire, à la limite, de remplir la promesse tant il devient clair que le journal n'est maintenu que pour être mal tenu, négligé: les «tas de choses à noter » (p. 320) peuvent bien s'accumuler à l'horizon, ils sont destinées à rester non notés, précisons: à être notés comme non notés, ce qui n'est pas tout à fait la même chose que de ne pas être notés du tout.

À parcourir les notes autoréflexives sur le processus même de la prise de notes qui obsèdent ici le diariste, on ne peut manquer de penser que cette forme d'écriture, qui soulève constamment par son insuffisance doutes et souffrance chez Mannoni, est la traduction, sur le plan esthétique, de la question de la sincérité qui hante ce journal. Comme Stendhal dans Henri Brulard auquel il s'identifie, Mannoni fait sans cesse " un effort pour être naturel " (p. 250): "Je pleurniche avec sincérité, écrit-il ailleurs à propos d'un rêve, mais avec le sentiment qu'on doit me trouver hypocrite " (p. 257)6. "Vous êtes un obsessionnel guéri», lui dira Lacan

6 Sur ce point aussi, les plaintea abondent, les oscillations entre désir et regret se font insistantes: "Que ce journal reste loin de la sincérité absolue. Telle page fut écrite avec l'idée qu'elle pourrait être lue. Tel autre avec le désir de la faire lire. II est déjà assez compromettant... Mais avec "sincérité absolue", je serais obligé de l'enfermer sans cesse à clé. Mais ça vaudrait la peine... " (ibid., p. 175; voir également p. $151,257,267,268,330,337,349$, etc.) 
au terme de son analyse. Comment ne pas voir que la pratique de la note a partie liée, en quelque point peu articulé, avec ce diagnostic? Si la sincérité, même (surtout?) "pour moi " seul, conduit le sujet vers l'infini - «[...] c'est à cela que j’ai reconnu que l'amour de la sincérité était un vice, parce qu'au-dessus de toute sincérité, qu'elle qu'elle soit, il y en a une plus grande» (p. 283) -, on comprendra qu'elle détermine, au moins partiellement, la difficulté grandissante de la notation elle-même:

Je n'écria pas assez dans ce journal. C'est que, pour noter exactement ce qui m'imtéresse, il faudrait une patience et un temps que je n'ai pas. Résumé, tout événement est sans intérêt. Dans son «texte intégral ", il demanderait des heures, et peut-être des jours, de la mémoire, un arrêt du mouvement de la vie, une grande capacité de discernement rétrospectif " (p. 324).

Où trouver ce temps «pour noter exactement ce qui m'intéresse", où arrêter durant des heures le mouvement de la vie, sinon dans l'espace analytique lui-même?

Au fait, se demandera-t-on, qu'en est-il du fameux cahier de l'analyse de Lacan? Eh bien, le lecteur qui en attendait des révélations fracassantes sera amèrement déçu: le journal se fait sur cet épisode, encarté en son cœur mais à part, en aparté presque, singulièrement laconique. Et c'est ce silence même qui se fait le plus parlant quant à l'importance de cet événement, véritable point tournant inscrit en creux entre deux carnets. En dépit de la décision de noter à part, sur «le nouveau cahier", tout ce qui se rapporte à "l'analyse que le $D^{r}$ Lacan a commencée hier» (p. 272), il devient rapidement évident que rien des séances ne pourra être noté, depuis l'interrogation inquiète des premières résistances ( Comment se fait-il que je ne puisse pas tenir un journal suivi de ma psychanalyse? Scotomisation, comme dit Lacan? Vraisemblable, mais la raison? À aborder dans l'analyse elle-même, je crois " [p. 281], jusqu'à l'inscription de l'effacement pour manque de sujet ( Je ne note pas volontiers les séances parce qu'il ne s'y produit rien de sensationnel [p. 303]), puis à cause de l'interdit de l'analyste lui-même, que Mannoni accepte avec soulagement ("Mais Lacan me conseille de ne pas tenir un journal de ces séances. J'arrête donc, avec plaisir d'ailleurs. Je n'aimais pas le tenir " [ibid.). Devant ce blanc qui recouvre l'espace analytique, nulle surprise cependant pour le lecteur 
qui aura reconnu là la stratégie familière de la note réitérant qu'il n'y a rien à noter, qu'ici rien n'aura eu lieu que le lieu. Ce n'est que plus tard que les effets de l'analyse se feront sentir dans la prise de note elle-même, de plus en plus réservée à la transcription des rêves. Comme l'écrit Mannoni au moment où il ne croit plus avoir besoin de son journal comme "instrument d'auto-analyse", " [j]e suis prêt non pas à reconstruire quoi que ce soit, mais à commencer n'importe quoi... Avec quel esprit de suite? (p. 331). Renoncer à la reconstruction, commencer n'importe quoi sans se soucier de la suite, ne serait-ce pas en effet aborder une autre pratique de la note, mieux accordée à la leçon de l'analyse, où il s'agirait moins de tenter une impossible restauration du sujet que de le rendre à son vide? C'est à cette condition, semble-t-il, que suspendre dès lors « in nugis", la pratique du "journal suivi» cesse d'être une forme de résistance à l'analyse et que la note redevient ici une écriture totalement accueillante, signe d'un affranchissement et d'une liberté nouvelle du sujet

$$
\text { *** * }
$$

Vincent Kaufmann mentionne de manière rapide dans L'équivoque épistolaire une hypothèse suggestive à propos des écrivains-épistoliers, proposition qui «peut paraitre excessive ", prévient-il (elle mène de fait la réflexion assez loin du point de vue des échanges entre genres): «Il y a ceux, écrit-il, qui conçoivent leurs lettres comme une pratique littéraire, et ceux qui font au contraire de leurs textes littéraires l'équivalent d'une lettre " ${ }^{7}$. Ce renversement des proportions entre l'œuvre et la note, les modes majeur et mineur, le monument et les miettes, on pourrait le transposer en pensant au cas d'un autre écrivain qui a pratiqué la note de manière constante tout au cours de sa vie d'écrivain, mais sur un mode sec et lapidaire, aux antipodes de celui adopté par Mannoni: il s'agit des "Réflexions " d'Elias Canetti. Je m'en tiendrai ici au dernier ouvrage paru, Le cour secret de l'horloge ${ }^{8}$, qui prolonge les réflexions de la période 1942-1972, recueillies dans Le territoire de l'homme.

7 Vincent Kaufmann, op. cit., p. 170.

8 Elias Canetti, Le cceur secret de l'horloge. Réflexions 1973-1985, traduit de l'allemand par Walter Weideli,coll. "Les grandes traductions ", Paris, Albin Michel, 1989, 234 p. Les références seront indiquées directement dans le texte: sauf indication contraire, c'est Canetti qui souligne. 
On observe en effet chez Canetti une économie textuelle assez particulière, se partageant entre les grandes œuvres (les trois volumes d'Histoire d'une vie, et surtout peut-être le magistral Masse et puissance) et la pratique d'une écriture fragmentaire (brefs essais, aphorismes, notes et réflexions), où il réfracte ces mêmes interrogations, mais en utilisant le mode plus intime du journal. Il faut cependant préciser que ces termes - intimité, journal, quotidien - prennent ici, on le verra, un sens beaucoup plus radical, assez étranger à leur acception courante.

Sur un plan strictement matériel, les réflexions réunies dans Le cour de l'horloge sont présentées selon le cours des ans, de 1973 à 1985, sans autres annotations de lieu, d'heure, d'humeur ou de circonstances. Sauf exceptions ( 1980 » et «1982 ", où figurent des essais un peu plus longs), les fragments et aphorismes n'occupent en moyenne pas plus d'une quinzaine de pages pour chaque année. On entrevoit peutêtre déjà à quel point le sujet de ces notes - qui éprouve, incidemment, le même amour que Mannoni pour Stendhal: «[...] j'admire Stendhal, non comme un modèle, mais comme une sorte de moi en mieux que je ne réussirai jamais, ne fûtce qu'un instant, à devenir vraiment. [...] Il est rapide, prend énormément de notes, les oublie. Je pensais autrefois faire de même" (p. 21) - est éloigné d'une conception romantique de l'introspection du moi. Nul épanchement chez Canetti, qui cherche par tous les moyens à échapper à la narration lisse du biographe, nulle effusion: une méfiance envers toute pensée de la fusion ( EEncore et toujours son esprit s'épuise en attouchements. Encore et toujours son esprit répugne à la fusion [p. 229]), un mouvement constant vers la séparation, la disjonction, la pensée dans sa blessure, d'amour-propre pour commencer: "Quand tout, dans une chose, s'emboîte parfaitement comme chez les philosophes, cela ne signifie plus rien. Disjoint, cela blesse et compte» (p. 190). Autrement dit, l'esprit de système, la totalisation empêchent de penser; seule la forme cassée, coupante comme un éclat, ébranle le sujet à partir du surgissement inattendu et indéterminé d'un " cela "

Ainsi, pratiquant la rupture sous toutes formes, Canetti commencera de fait par rompre avec la loi implicite du journal: le "nulla dies sine linea" devient ici un "Ne laisse pas s'écouler de jours sans signes. Quelqu'un en aura besoin " (p. 216). La transformation - une simple traduction qui adapte la sentence latine - pourra paraître minime à première 
vue. Il n'en est rien, puisque Canetti déplace de fait la destination de ces notes: le dépôt et la conservation pour mémoire "pour moi", habituellement confiés à ce genre d'écriture autobiographique - au sens fort du terme décornposé en " autos" (même), "bios" (vie) et graphie -, sont ainsi assignés à "quelqu'un d'autre». Ainsi, la "ligne» se change en "signe» pour l'autre, elle se fait ici littéralement appel de note.

Ce n'est là qu'une des transformations que Canetti impose au journal (ailleurs, il souhaitera précisément «un journal transformable, toujours le même" [p. 41]). Car qu'est-ce qu'un journal, sinon une pratique devenue régulière ${ }^{9}$ (relativement, tout au moins) de la note, c'est-à-dire l'inscription au jour le jour de ce jour même? Le lecteur ne retrouvera ici rien de tel, sauf en quelques rares épiphanies comme celle-ci: "Un soir de peine et de piment, devant la fenêtre, un héron» (p. 160), ou encore cette autre: "Des sons de piano provenant du platane» (p. 160), qui font d'ailleurs l'effet d'anamnèses, temps deux fois perdu plutôt que retrouvé. Chez Canetti, le temps sera compté selon d'autres mesures que celles du jour, de la semaine, du mois ou même de la saison («Les journées sont devenues des gouttes, elles tombent une à une, plus rien ne converge, l'année n'est plus qu'un verre à demi plein " [p. 66]). C'est à l'éternité, rien de moins, que devront pouvoir s'affronter les pensées de ce "testament en miettes " (Canetti reprend ici la célèbre formule de Nietzsche). "Plus que tout, tu désires - quelle modestie! - une éternité de lecture" (p. 18). Je reviendrai plus loin sur ce motif essentiel qui lie la note et la lecture, mais on remarquera pour l'instant que la note, lapidaire par son ton, l'est également par sa fidélité à l'étymologie, à travers ce désir de laisser trace sur la pierre. Soulignant le paradoxe d'une régularité discontinue au cour de l'écriture du journal, Canetti interroge ce mode d'inscription étrange, qui laisse une trace mais par usure, par érosion de la forme. Le dispositif thanatographique de la note,

9 Roland Barthes évoquait ce paradoxe dans le fragment intitulé " Du fragment au journal ": "Sous l'alibi de la dissertation détruite, on en vient à la pratique régulière du fragment; puis du fragment, on glisse au "journal". Dès lors le but de tout ceci n'est-il pas de se donner le droit d'écrire un "journal " ? " [puisque] "le "journal " (autobiographique) est cependant, aujourd'hui, discrédité" (Roland Barthes par Roland Barthes, coll. "Écrivains de toujours", Paris, Seuil, 1975, p. 99). 
sa destination posthume, son adresse d'outre-tombe aux lecteurs fantômes, sont encore accentués du fait que la forme de ces fragments est étroitement reliée à la vieillesse ellemême: ces notes sont avant tout "[u]ne ouvre de vieillesse sous forme de lettres» (p. 197). Loin d'exercer une fonction mnémotechnique, la note vise ultimement l'oubli: «Avec l'affaiblissement de la mémoire, tout ce qu'on a découvert par soi-même se détache de soi " (p. 166).

Il s'agira bien, dans ces circonstances, de prendre des notes pour les oublier: Canetti se révèle, tout compte fait, plus près de Stendhal qu'il ne le pensait. Avec la notefragment, forme affaiblie, affinée du soi se détachant de luimême, comme la pierre rendue poreuse sous l'action de l'eau, le travail, d'inscription de ces notes est souvent au plus près du travail du deuil, amorçant cette seconde - et plus difficile - phase d'oubli qui suit la remémoration-incorporation de l'objet perdu. D'où peut-être ces parcours souvent inversés chez Canetti, par lesquels il remonte le cours d'une mémoire non seulement personnelle, mais également phylogénétique. Nombre de ces réflexions tournent en effet, telle l'«alouette d'idées " (p. 229), autour d'une rétractation de l'homme vers l'animal: " "Homme" n'a plus rien d'étonnant pour lui. Ce qui l'étonne, c'est "animal" " (p. 223), "[i]l a besoin des diverses formes d'animaux pour ne pas désespérer de toute forme" (p. 221). De manière analogue, on pourrait interpréter en ce sens la remontée vers l'oralité présente dans ces fragments sous plusieurs formes - stade oral, mais aussi naissance de la narration -, la vieillesse étant cet âge de la vie qui opère la traversée en amont des couches psychiques antérieures du sujet et délie ses identités.

Ainsi, de manière générale, les notes de Canetti frappent d'abord par leur tranchant, on dirait mieux leur mordant ${ }^{10}$, tant les affects du ressentiment, de la rage, voire de la méchanceté «Nostalgie de la haine» (p. 215), lâche-t-il, les dents serrées

10 Voir, par exemple, les notes suivantes: "La seule chose à ne pas se venger de lui, ce sont des réflexions " (p. 18); « ll cherche des phrases que nul n'a encore mâchées " (p. 62); "Il ne veut plus de pensées qui mordent: il veut des pensées qui soulagent la respiration" (p. 81); "Ne plus chercher à mordre: laisser aux phrases la bouche ouverte" (p. 178). On remarquera que la note s'allie ici à la question de l'oralité, et de façon plus générale, aux rapports aux langues. Sur ce motif de la "langue coupée" chez Canetti, on se reportera au bel article de Michel Schneider, "Elias Canetti: la défusion des langues", Le temps de la réflexion, Paris, II, 1981, p. 384-402. 
presque, à la fin du volume - se font insistants ici et ne sont que très lentement dissous au fil du temps. De fait, même en une douzaine d'années, la lecture de soi déposée dans ces fragments subit l'épreuve du temps, le « soi * devenant plus friable. «Il rompt avec lui-même et pousse un soupir de soulagement. Il ne veut plus jamais rien savoir de lui-même" ( $p$. 235), écrit Canetti dans le tout dernier fragment: on peut penser que, rendu en ce point limite, la lecture que le sujet faisait de luimême a non seulement été soumise à l'épreuve du temps, mais que le temps lui-même a subi l'épreuve de la lecture ${ }^{11}$.

Mais cette épreuve du temps, c'est d'abord la phrase ellemême qui doit l'affronter. Souvent condensée comme un cristal, durement repliée autour d'un noyau (Canetti adopte souvent la forme nominale), elle est non seulement minimaliste, elle cherche toujours davantage à couper court ("Plus bref, plus bref, jusqu'à ce qu'il ne subsiste qu'une unique syllabe par laquelle tout est dit» [p. 67]), à se diminuer ("À présent, les grands mots te font défaut à toi aussi: combien t'en reste-t-il de petits?» [p. 205]), à dire le plus par le moins («Ce que tu n'as pas dit s'améliore" (p. 204), bref, à réduire son expression en la soumettant à la loi de la plus grande concision ("Tu n'as encore jamais réussi à être aussi bref que tu voulais l'être" [p. 216]), au point qu'on pourrait parler ici de constriction, un mouvement d'écriture alliant la contrainte, la restriction, la rétractation ( IIl n'inventait plus guère de phrases que pour en rétracter d'anciennes"), le resserrement aussi, en une construction qu'on sent mortelle (ce n'est pas un hasard si Canetti s'adresse directement à la mort dans plusieurs fragments). Sous la coupe de la "Terreur du fragmentaire" (p. 233), la note de Canetti se coupe elle-même la parole, elle se donne souvent comme une machine à faire le vide autour d'elle ("Il parle sans relâche jusqu'à ce que tout s'effondre» [p. 204]), ou en elle-même, comme dans cette petite fable proche de l'esprit du haïku: «Il rentra chez lui. Rien n'avait bougé. La table s'était dissoute. Il s'assit et se mit à écrire dans le vide " (p. 195). La note devient ici le lieu d'éradication du sujet, ces fragments les "Derniers spasmes dans l'écriture" (p. 62) où il veut «[s]'éteindre dans la plus courte des phrases» (p. 118). Par cette concision extrême, les notes de Canetti rompent littéralement le sujet biographique qui écrivait un journal pour se

11 J'emprunte cette idée à Jean-Michel Rey, Les enfants du silence, coll. " Carnets ", Paris, Plon, 1990, p. 121. 
comprendre («Plus concis, toujours plus concis, jusqu’à ce qu’il ne se compresse plus lui-même» [p. 106]): le journal, loin de totaliser une existence, est le lieu où «[i]l se rappelle tout le non-vécu" (p. 174).

Or, se demandera-t-on avec Jean-Michel Rey, devant tant de déliaison à l'œuvre dans ces notes, «[q]u'est-ce qui peut faire le lien, chez un même sujet, entre ses différentes lectures décousues? " 12 Quoi, sinon la lecture elle-même? Car comment expliquer que la lecture de notes ayant pour motifs explicites la dissolution du sujet, ses deuil et mélancolie, l'oubli, voire la notation du Rien dans sa plus forte négativité, produise l'effet contraire et soit constamment stimulante, régénérante même, pour le lecteur? C'est qu'il y va dans la poétique de la note de ce qui fait l'essentiel du rapport du lecteur à la chose lue: la note est une expérience très révélatrice de l'acte de lecture luimême, sa variante et ses variations, si l'on peut dire. Ainsi, certain lecteur de ma connaissance ne jure que par les footnotes, un autre ne supporte pas les coups de crayon dans les marges, un autre encore aime bien par ces griffonnages avoir accès à la lecture la plus intime, saisie au vif. "Sur certains livres, note Rey dans Les enfants du silence, ces coups de crayon qui ressemblent à une oreille " ${ }^{13}$. Valéry portera pour sa part un coup fatal à cette tentative de typologie lorsque détachant la note de son support matériel même, il en décrira la forme extrême, échappant à toute forme et à tout lieu textuel précis, en ces termes:

J'ai passé ma vie à noter (non dans les marges de mes livres et presque jamais à propos de lectures), mais à noter ce qui me venait à l'esprit. [...] La lecture attentive n'est qu'un commentaire continu, une succesion de notes échappées à la voix intérieure. Les notes marginales sont une partie des notes purement pensées. ${ }^{14}$

12 Jean-Michel Rey, op. cit., p. 158. Sur l'ouvrage de Rey, voir mon compte rendu ("La lecture et ses dispositions", Spirale, Montréal, $n^{\circ} 102$, décembrejanvier 1991, p. 7), où je soulignais que « [l]a théorie de la lecture est peut-être plus intimement questionnée qu'on ne le croit par cette poétique de la note, elle-même à la limite non théorisable, ou remettant en mouvement les limites de la théorie."

13 Ibid., p. 100.

14 Paul Valéry, cité par Jean-Michel Rey, op. cit., p. 182-183. 
82

Ainsi, à la limite, tout peut devenir note: pure pensée, la note est partout et nulle part, elle traverse toutes les catégories et tous les genres, elle déborde les marges et le bas des pages qui lui étaient réservées...

Mais revenons tout de même, pour finir, au genre de notes qui nous intéresse tout particulièrement ici, la note de lecture. «Sans lectures, constatait Canetti avec justesse, plus rien ne vient à l'esprit. Rien ne se rattache à rien. Tout reste isolé et flottant. Une étendue de tiges clairsemées, et non drues dans l'herbe" (p. 169). On ne saurait mieux suggérer que la lecture, selon son étymologie, cueille les riens et les rattache, les lie en une gerbe d'idées ou d'associations qui viennent alors librement à l'esprit. Entre la note et la lecture, il y a de nombreuses affinités électives, leurs rapports relèvent de fait tellement de l'évidence qu'ils passent presque inaperçus. L'idée même de tenir un journal ou de remplir des carnets est inséparable de la note de lecture. Ces dernières années, un éditeur, Plon, est allé jusqu'à consacrer à ces marginalia une nouvelle collection intitulée "Carnets" où, précisément, il a publié les notes d'une quinzaine d'écrivains (ces quelques titres - Carnet de nuit [Philippe Sollers], Le jour et l'heure [Marcelin Pleynet], Au jour le jour [Michel Butor], Compté, pesé, divisé [Henri Thomas], Mémoires d'un dictaphone [Jean-Claude Gallota] donnent une bonne idée du programme de la collection). Parmi les titres déjà parus, on retiendra celui de Rey, Les enfants du silence - sans doute une œuvre de commande en raison de la période fort courte couverte par l'annotation - , un recueil de notes de lecture où il fait justement retour à plusieurs reprises sur les liens qu'entretient la note avec le procès de la lecture. Prenant pour point de départ les diverses positions, mieux, les dispositions, où joue chaque fois un problème différent d'accommodation, d'ajustement pour le lecteur - parmi les lectures répertoriées, mentionnons les lectures décousues, simultanées, croisées, par superposition, surimpression, à courte ou longue vue, lentes ou rapides, myopes ou presbytes, etc. -, Jean-Michel Rey met l'accent sur les notes comme «innombrables dépôts de lecture, des alluvions» qui finissent par former "notre géologie propre, ces matières précaires, friables, qui à la longue se solidifient en nous " ${ }^{15}$. Il souligne 
également l'importance de la relecture où la note - via les passages soulignés ou recopiés, jamais les mêmes d'une fois à l'autre - n'est pas à sa place, change de place selon des stratégies de "remise, délai, sursis, ajournement, crédit, expectative». Il fait surtout la preuve, par un certain usage de notes elles-mêmes rédigées au jour le jour, que la note de lecture n'est pas exclusivement soumise à un principe d'utilité. Sa fonction, on l'a vu, n'est pas d'abord et avant tout de servir d'aide-mémoire ("Citations oublieuses", disait Canetti). Elle n'est pas davantage au service d'une démonstration ou d'une thèse qui doivent se déployer plus tard, le "travail d'application d'une pensée déjà formée - supposée telle - nécessairement rempli de formules, voué à la platitude "16. Autrement dit, en dépit de préjugés qui tentent toujours de la limiter, il ne s'agit pour la note de lecture ni d'étudier, ni d'expliquer, ni de développer (elle serait même plutôt de ce point de vue un enveloppement, selon la belle formule de Canetti: "Nombre minime de pensées au cours d'une existence; leur retour constant comme si elles étaient neuves et néanmoins familières, enveloppées dans le temps comme dans des feuilles» [p. 214]).

La note de lecture reste donc au contraire un commentaire "libre", un "excès" de lecture ${ }^{17}$, une outrance aussi (voir par exemple la gêne qui surgit devant certaines annotations anciennes). Elle est une bribe, un bris de lecture, un ex-libris; une manière, comme l'écrit Rey, de dire et de lire " avec un minimum: des riens qui indéfiniment se recoupent" 18 , dans une sorte de relance indéfiniment ouverte sur le vide. Elle est l'exercice de lecture dans toute sa nudité, travail à la fois élémentaire (il y a de l'ascèse, de la rigueur, de la gymnastique dans la note de lecture, pose et figure de la pensée en vol, "suspense qui fait le vide: une halte et un rebond "19) et dépensier, mené en pure perte (le tourbillon des références, la circulation des noms propres multipliés à l'infini, par exemple). Mais au-delà de ces traits, la note de lecture reste une pratique exemplaire pour deux raisons essentielles, qui sont le versant l'une de l'autre, points de vue du lecteur et de l'écrivain échangeant leur place: pour le

17 Ibid., p. 72.

18 Ibid., p. 19.

19 Ibid., p. 165. 
84

premier, les notes de lecture sont * de simples pierres d'attente, des jalons en vue de ce que nous ne connaissons pas - de ces choses dont nous ne savons pas que nous les cherchons " 20 , pour le second, elles renvoient à * [1]a tâche modeste de l'écrivain, [qui] est peut-être finalement la plus importante: la transmission des choses lues ${ }^{21}$. 